\title{
A characteristic continuous wave Doppler signal in cor triatriatum?
}

\author{
M Alwi, Z A A Hamid, R Zambahari
}

\begin{abstract}
Continuous wave Doppler recordings of the turbulent jet through the restrictive orifice of a left atrial partition in a patient with corrected transposition of the great arteries and cor triatriatum showed alternate bands of high intensity diastolic and low intensity systolic signals with preservation of the normal configuration of the diastolic $E$ and $A$ peaks. It is thought that Doppler studies in cor triatriatum will provide useful complementary haemodynamic information in the echocardiographic diagnosis of this anomaly.
\end{abstract}

Cor triatriatum can be diagnosed by cross sectional echocardiography. Because this is a rare condition there are few reports of the diagnosis of cor triatriatum by Doppler echocardiography. We report a case of congenitally corrected transposition of the great arteries with cor triatriatum and an unusual continuous wave Doppler signal from the turbulent flow through the orifice of the left atrial partition.

\section{Case report}

A four year old boy who had been treated for asthma was referred because of a heart murmur. He had mild exercise intolerance. On clinical examination he was comfortable at rest and there was no cyanosis. His pulse rate was 105 beats $/ \mathrm{min}$. There was a precordial bulge and grade $3 / 6$ systolic and diastolic murmurs were heard over the apex. The chest radiograph showed situs solitus, levocardia, and mild cardiomegaly. The appearance of the lung fields was suggestive of pulmonary venous obstruction with perihilar congestion and fluid in the horizontal fissure. The electrocardiogram showed sinus rhythm, an axis of $-20^{\circ}$, and left atrial enlargement. Cross sectional, colour flow mapping and continuous wave Doppler examination was performed with a Toshiba SSH-160A. The descending aorta, inferior caval vein, and abdominal viscera were normally lateralised. There were atrioventricular and ventriculoarterial discordant connections without an associated ventricular septal defect, pulmonary stenosis, or tricuspid regurgitation. The peak pulmonary velocity was $1.4 \mathrm{~m} / \mathrm{s}$. The pulmonary arteries were dilated. Additionally, a partitioning membrane in the left atrium was seen and distended pulmonary veins entered the prox-
Department of Cardiology, General Hospital, Kuala Lumpur, Malaysia M Alwi

Z A A Hamid

R Zambahari

Correspondence to Dr Mazeni Alwi, The General Hospital, 50586 Jalan Pahang, Kual Lumpur,

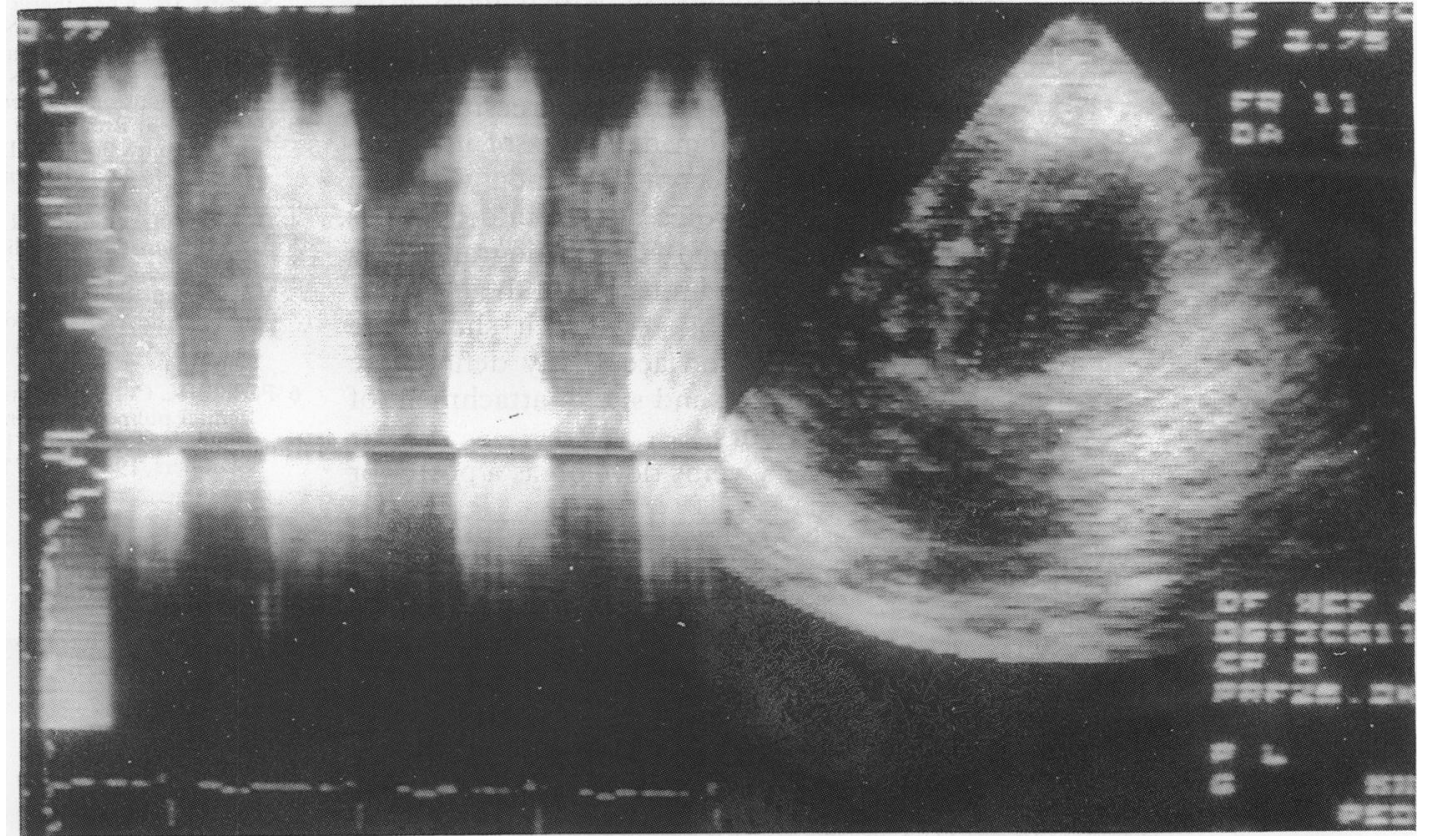

Figure 1 Continuous wave Doppler signal of flow through the membrane orifice showing alternate bands of high intensity diastolic and low intensity systolic signals with normal $E$ and $A$ peaks. Peak velocities were high in both parts of the cardiac cycle (upper limit of velocity scale is $+3.77 \mathrm{~m} / \mathrm{s}$ ). 

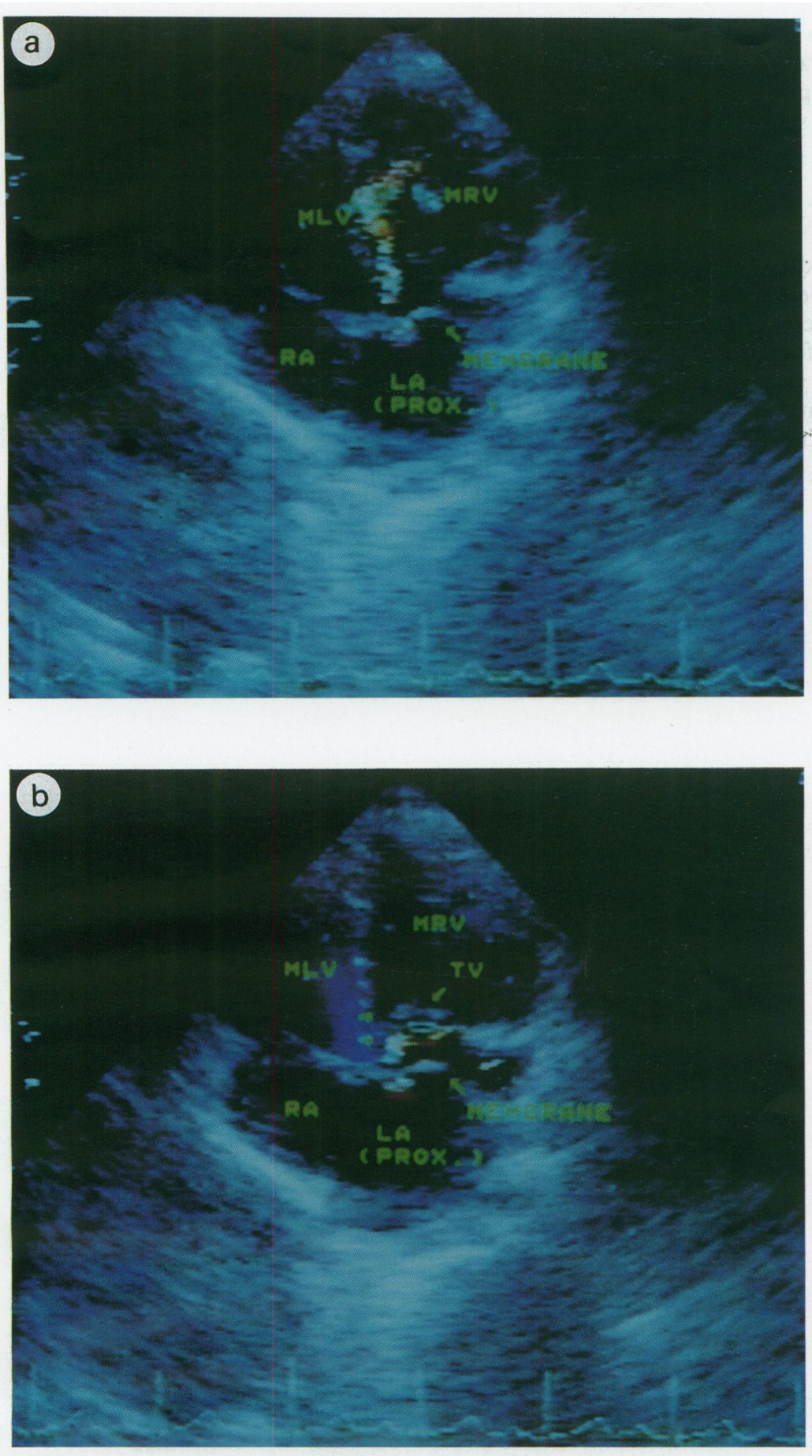

Figure 2 Colour flow mapping showing $(A)$ the diastolic frame with a turbulent jet through the restrictive membrane orifice and $(B)$ the systolic frame showing attenuated flow through the orifice against a closed atrioventricular valve. Note the more apical setting of the atrioventricular (morphologically tricuspid) valve (double arrows). $L A$ (prox), proximal left atrial chamber; $M R V$, morphologically right ventricle, $M L V$, morphologically left ventricle; $R A$, right atrium; $T V$, tricuspid valve.

imal chamber. This membrane was attached some distance away from the annulus of the atrioventricular valve. Colour flow mapping showed a turbulent jet arising from a very small eccentric orifice in the partitioning membrane close to the interatrial septum.

Continuous wave Doppler interrogation of the turbulent jet through the orifice showed an unusual spectral display. There were alternate bands of high intensity diastolic and low intensity systolic signals in a positive direction producing a vertically striped appearance.
The peak velocities were abnormally high in both parts of the cardiac cycle. The velocity of the low intensity systolic signals reached a minimum $(1.8 \mathrm{~m} / \mathrm{s})$ in early systole and gradually peaked $(2.4 \mathrm{~m} / \mathrm{s})$ at end systole (a mean of three consecutive beats). In the high intensity diastolic signals the normal $\mathrm{E}$ and $\mathrm{A}$ peaks were preserved despite a high peak velocity of $2.8 \mathrm{~m} / \mathrm{s}$. The passive phase of ventricular filling in diastole was not prolonged (fig 1).

We compared flows through the membrane orifice in both parts of the cardiac cycle on colour flow mapping by means of the cine-loop facility and electrocardiographic marker to identify systolic and diastolic frames. Continued forward flow through the orifice, though attenuated, was seen during systole (fig 2 ). The diagnosis was confirmed at surgery and the membrane was excised. Postoperatively the spectral display of flow through the left atrioventricular valve was normal, resembling that of the right atrioventricular valve.

\section{Discussion}

Though the coexistence of cor triatriatum with corrected transposition of the great arteries is rare it has been reported before. ${ }^{1}$ Because cor triatriatum can be readily diagnosed by cross sectional echocardiography cardiac catheterisation is not usually needed before operation. ${ }^{2}$

Because the orifice in the partitioning membrane is usually very small and restrictive of flow, Doppler echocardiography was expected to provide haemodynamic information that complemented the anatomical details obtained by cross sectional echocardiography. However, there are few reports on this subject because the condition is so rare. Previous reports in English merely mentioned high velocity diastolic signals when the pulsed Doppler sample volume was placed beneath the restrictive orifice. ${ }^{34}$

Several of the features of the signals recorded in our patient during continuous wave Doppler examination had not been described before. Firstly, by using the cine-loop facility and analysing the colour flow images frame by frame we were able to demonstrate continued forward flow through the orifice of the membrane during the phase when the morphologically tricuspid (left atrioventricular) valve was closed in systole. We suggest that this was the mechanism responsible for the low intensity positive signals on the spectral display: pressure in the distal left atrial chamber would be expected to remain lower than that in the proximal chamber and hence cause a continuous forward flow. These positive systolic signals have been described before in a report in Spanish. ${ }^{5}$ Secondly, despite the high velocity in diastole, the $E$ and $A$ peaks were normal, suggesting normal mid-diastolic closure of the atrioventricular valve with no prolongation of the phase of passive ventricular filling and that the obstruction is not likely to be at the level of the valve. These spectral display findings were not seen in 
severe obstruction of the left ventricular inflow tract by a stenotic mitral valve. ${ }^{6}$ Supravalvar mitral rings may pathophysiologically mimic cor triatriatum but they seldom occur in isolation and are commonly associated with congenital mitral stenosis. ${ }^{7}$ We do not know of any reports of Doppler examination in congenital mitral stenosis but, as in acquired mitral stenosis, we would not expect a positive systolic signal on the spectral display because the pressures in the left ventricle and left atrium would prevent forward flow through the mitral valve during systole. It remains to be seen whether the features we describe are characteristic of cor triatriatum.

We thank Mrs Zahiyah Bte Abdul Rahman and $\mathrm{Mr}$ Kamaruzaman Bin Othman.
1 Marin-Garcia J, Tandon R, Lucas RV, Edwards JE. Cor triatriatum: study of 20 cases. Am J Cardiol 1975;35 $59-66$.

2 Östman-Smith I, Silverman NH, Oldershaw P, Lincoln C, Shinebourne EA. Cor triatriatum sinistrum. Diagnosti features on cross sectional echocardiography. Br Heart 1984;51:211-9.

3 Vick WG, Murphy DJ, Ludomirsky, et al. Pulmonary venous and systemic ventricular inflow obstruction in patients with congenital heart disease. Detection by com bined two dimensional and Doppler echocardiography. J Am Coll Cardiol 1987;9:580-6.

4 Radhakrishnan S, Shrivastava S. Doppler echocardiography in the diagnosis of divided left atrium (cor triatriatum sinister). Ind J Cardiol 1988;21(2):180-3.

5 Rodriguez Garcia MA, Suarez Fernandez G, Perez Lorente F, Santamanrta Diez N. Doppler echocardiography in cor triatriatum: Apropos of 2 cases. Rev Esp Cardiol 1989;42 triatriatum: Apropos

6 Mayala APG, Roelandt J. Mitral valve disease. In: Houston AB, Simpson IA, eds. Cardiac Doppler ultrasound: A clinical perspective. London: Wright, 1988:57-68.

7 Anderson RH, MacCartney FJ, Shinebourne EA, Tynan M. eds. Paediatric Cardiology. Edinburgh: Churchill Livingstone, 1987:1023. 